\title{
Anabases
}

ANABASES Traditions et réceptions de l'Antiquité

$15 \mid 2012$

Varia

\section{MOnica BERTI et Virgilio COSTA, La Biblioteca di Alessandria. Storia di un paradiso perduto}

Ilaria Sforza

\section{OpenEdition}

\section{Journals}

Edizione digitale

URL: http://journals.openedition.org/anabases/3808

DOI: 10.4000/anabases.3808

ISSN: 2256-9421

Editore

E.R.A.S.M.E.

\section{Edizione cartacea}

Data di pubblicazione: 1 aprile 2012

Paginazione: 244-246

ISSN: 1774-4296

Notizia bibliografica digitale

Ilaria Sforza, « monica вERTı et Virgilio costa, La Biblioteca di Alessandria. Storia di un paradiso perduto », Anabases [Online], 15 | 2012, Messo online il 01 avril 2012, consultato il 21 septembre 2020. URL http://journals.openedition.org/anabases/3808 ; DOI : https://doi.org/10.4000/anabases.3808

Questo documento è stato generato automaticamente il 21 settembre 2020.

(c) Anabases 


\title{
MOnica BERTI et Virgilio COSTA, La Biblioteca di Alessandria. Storia di un paradiso perduto
}

\author{
Ilaria Sforza
}

\section{NOTIZIA}

Monica BERTI et Virgilio costA, La Biblioteca di Alessandria. Storia di un paradiso perduto, Tivoli (Rome), Edizioni Tored, 2010, 279 p. + Xvi p.

30 euros / ISBN 978-88-88617-34-3.

1 È un saggio di piacevole lettura e ben documentato sulla storia della biblioteca di Alessandria, dalla fondazione della città da parte di Alessandro durante la sua spedizione in Egitto nel 333 a. C., fino alla distruzione del Bruchion, il quartiere in cui si trovavano gli edifici del Museo, sotto Aureliano nel 273 d. C.

2 Nei primi quattro capitoli gli autori - Monica Berti nei primi due, Virgilio Costa nei successivi - ripercorrono la vita del complesso alessandrino in cui sorgeva il Museo fino alla sua progressiva perdita di importanza e alla distruzione materiale, avvenuta almeno in parte già con l'incendio appiccato da Cesare ad alcuni edifici adiacenti al porto nel 48 a. C. quando dovette difendersi dalle truppe dello stratega egiziano Achillas. Il quinto capitolo, scritto in collaborazione, riprende e sviluppa l'idea ispiratrice del saggio, quella di mettere in luce la «relazione ideale tra la biblioteca di Alessandria e le odierne "biblioteche universali"» (p. Ix). I due studiosi, infatti, dirigono nell'ambito della Scaife Digital Library un progetto di catalogazione di tutte le fonti sul Museo e sulla Grande Biblioteca di Alessandria (www.alexandrianlibrary.org) e Monica Berti dirige il sito web Fragmentary Texts. Collecting and Representing Quotations of Lost Authors and Works (www.fragmentarytexts.org), interamente dedicato alle nuove iniziative di raccolta e digitalizzazione delle fonti antiche. 
3 Se aveva ragione Plutarco (Vita di Alessandro 26, 10-11) nell'affermare che Alessandro fondò la celebre città egizia che porta il suo nome ispirato da un verso dell'odissea (IV, 354-355) che ci descrive l'isola di Faro («Un'isola c'è nel mare flutti infiniti, / davanti all'Egitto, la chiamano Faro») non possiamo saperlo, ma di certo il grande condottiero macedone non immaginava che presso gli edifici adiacenti al Porto Grande sarebbe sorto di lì a poco un complesso museale in grado di ospitare, secondo le stime più contenute, oltre ventimila rotoli di papiro. Ma quando ebbe inizio il grandioso progetto di «raccogliere, là dove possibile, tutti i libri del mondo», come riferisce l'autore della cosiddetta Lettera di Aristea a Filocrate (§ 9) e, soprattutto, chi portò avanti questo ambizioso progetto?

Dopo avere seguito, nel primo capitolo, l'accurata descrizione di Strabone (XVII, 1, 8-10) del territorio dove sorgeva e in particolare degli edifici ospitati all'interno della città di Alessandria, Monica Berti prende in esame, nel secondo capitolo, una serie di fonti che testimoniano dell'ideazione del progetto culturale patrocinato dai sovrani ellenistici Tolemeo I Soter e Tolemeo II Filadelfo, grazie alla fondamentale mediazione del filosofo peripatetico e uomo politico greco Demetrio Falereo, di acquistare, trascrivere e tradurre, ove fosse necessario, come nel celebre caso del Pentateuco, tutte le opere della letteratura greca e le più importanti opere straniere reperibili. La fondazione e lo sviluppo della Biblioteca di Alessandria, inoltre, coinvolsero certamente più di una generazione, come suggerisce un aneddoto riferito a Tolemeo III Euergete tramandatoci da Galeno (In Hippocr. librum iii epidemiarum 17a, 606-607 Kühn), secondo cui questi ambiva «così tanto a possedere libri che ordinò di fargli avere anche quelli che si trovavano su tutte le navi che entravano nel porto»; gli originali, le cui copie erano restituite ai proprietari, andavano a costituire un fondo a parte che recava la scritta «dalle navi» ('́k $\left.\pi \lambda \circ i_{\omega} \omega v\right)$.

5 Ma come si svolgeva la vita degli studiosi alessandrini operanti nel Museo? Essi erano davvero come uccelli rari racchiusi in una voliera, cinti da palizzate di libri e costantemente occupati a beccarsi l'un l'altro come ce li descrive Ateneo (Deipn. I 22d) riportando una testimonianza del silografo Timone di Fliunte? Stando alla descrizione di Strabone (XVII, 1, 8), il Museo era una struttura provvista di «una passeggiata ( $\pi \varepsilon ́$

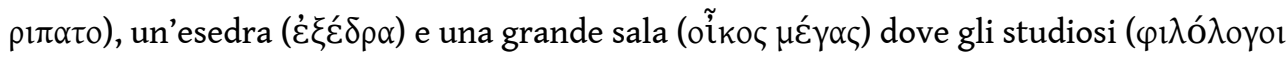

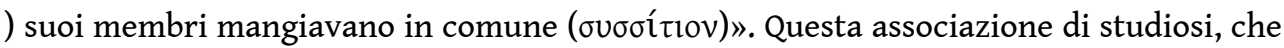
sopravvisse fino al I secolo a.C., disponeva di beni comunitari e un sacerdote responsabile del Museo era designato prima dal re, poi dall'imperatore. Si trattava pertanto di «strutture analoghe a quelle dell'Accademia di Platone e del Liceo di Aristotele» (p. 89). Gli autori mettono bene in evidenza la continuità tra il Liceo aristotelico e il Museo di Alessandria, non solo in riferimento a un'analogia delle strutture destinate al sinodo degli studiosi, ma anche all'organizzazione del sapere. Mettono inoltre l'accento sulla problematica definizione del concetto di «biblioteca», che traduce di volta in volta i vocaboli greci $\beta \imath \beta \lambda i ́ \alpha$ e $\beta \imath \beta \lambda_{\imath} \circ \theta \eta ́ k \eta$. Quest'ultimo «non si riferisce necessariamente a una costruzione o a un ambiente a sé dotato di tutti quei servizi che caratterizzano una moderna biblioteca. L'etimologia del vocabolo allude infatti alla "teca dei libri", e cioè allo scaffale che conteneva i rotoli di papiro, oltre che naturalmente alla collezione stess» (p. 99).

Il terzo capitolo, «Scienza e filologia nella Biblioteca di Alessandria», è interamente dedicato a quelle figure di studiosi poliedrici, ben lontani dalla frammentazione specialistica del sapere che caratterizza invece la nostra epoca, che diedero il loro 
contributo alla classificazione, conservazione e produzione della cultura alessandrina, a partire da Filita di Cos, operante ad Alessandria sotto Tolemeo I, del cui figlio, il futuro Filadelfo, fu precettore, e che diede un contributo fondamentale alla nascente filologia

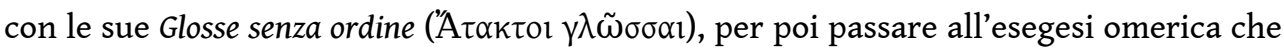
vede figure di spicco ad Alessandria come Zenodoto di Efeso, anche lui precettore del Filadelfo, Callimaco di Cirene, autore delle Tavole (Пívake) in cui classificava i principali autori della letteratura greca e le loro opere, Apollonio Rodio e il suo omonimo Apollonio Eidographos, Eratostene, che misurò la circonferenza terrestre in modo sorprendentemente esatto e ancora Aristofane di Bisanzio, che lavorò a una nuova diòrthosis di Omero, il grande Aristarco di Samotracia, l'ultimo direttore della biblioteca, e un suo indiretto epigono, Didimo di Alessandria, che fiorì nell'età di Cesare.

7 Ma cosa successe dopo Aristarco, cosa determinò la rovina del Museo? Fu la violenta repressione scatenata dall'Euergete II nel 144 a. C. per motivi di successione dinastica, cui seguì la diaspora di tutti quei filosofi, matematici, pittori, filologi che fino a quel momento avevano operato nel Museo e che in seguito, «ridotti dalla povertà a insegnare ciò che conoscevano, istruirono tanti uomini illustri» (Ath. Deipn., IV 184 c), o non fu piuttosto, quello, solo l'inizio di una più lenta erosione operata dall'interno, vale a dire dal tramonto di una cultura e dall'oblio delle vette di conoscenza che la condizione privilegiata degli intellettuali ospitati nel Museo aveva consentito?

Non è forse un caso che Lucio Russo nel celebre saggio La rivoluzione dimenticata (Feltrinelli, 1996) parlasse di rimozione della rivoluzione scientifica, ravvisando nella perdita della maggior parte delle opere degli intellettuali, scienziati, grammatici e filologi, che ne furono protagonisti una delle cause principali di tale rimozione: «La civiltà che, fra tante conquiste intellettuali ci ha tramandato anche l'idea stessa delle biblioteche e della gelosa conservazione del pensiero del passato è stata cancellata con le sue opere» (ibidem, p. 24).

9 Alcune possibili risposte a quest'ultima domanda che ci siamo posti - come muoiono le biblioteche - si trovano nel capitolo conclusivo de La Biblioteca di Alessandria: «Esse muoiono, ad esempio, quando viene meno l'impetus iniziale che ne aveva reso quasi necessaria l'esistenza [...]. Esse muoiono anche quando il contesto storico e i gusti del pubblico mutano» (p. 202). Un finale pessimistico? Non direi, poiché la risposta alla lenta e quasi inevitabile consun-zione dei rotoli di papiro e delle pagine di carta dei volumi custoditi nelle biblioteche odierne risiede senza dubbio - Monica Berti e Virgilio Costa ce lo suggeriscono - nelle biblioteche universali di oggi, quelle digitali, «immuni da ogni genere di fuoco, reale o metaforico» (p. 203). 


\section{AUTORI}

ILARIA SFORZA

Università degli Studi di Roma

"Tor Vergata"

kakridis@hotmail.com 